\title{
Über die Wirkung von Fermentinhibitoren auf INH-sensible und -resistente Zellen von Mycobacterium phlei
}

\author{
Von G. Mülller, H. Iwainsky und K. TäUfel \\ Aus dem Tuberkulose-Forschungsinstitut Berlin-Buch (Direktor: Dr. med. P. StrinBrück) \\ und dem Institut für Lebensmittelchemie und -technologie der Humboldt-Universität zu Berlin \\ (Direktor: Prof. Dr. Dr. h. c. K. TäUfeL) \\ (Z. Naturforschg. 15 b, 180-189 [1960]; eingegangen am 21. Dezember 1959)
}

\begin{abstract}
An Hand der Literatur wird ein kurzer Uberblick über die bisher bei Mykobakterien aufgefundenen Stoffwechselvorgänge bzw. die sie steuernden Fermente gegeben.

Mit Hilfe verschiedener Inhibitoren wird die endogene Atmung und der Stoffwechsel des Glycerins sowohl bei ruhenden als auch bei proliferierenden INH-sensiblen und -resistenten $M$. phlei vergleichend untersucht.

Auf Grund der Hemmung durch Fluorid, Jodacetat und Arsenit ist sowohl bei der endogenen als auch bei der Ruhe- und Vermehrungsatmung ein dem E m b d e n-M e y e r h of - Schema entsprechender Reaktionsverlauf anzunehmen.

Der weitere Abbau ist noch nicht geklärt. Es wird der sog. „Glyoxylsäurecyclus“ diskutiert. Mit Sicherheit wird die Beteiligung des Citronensäurecyclus bei ungehinderten Vermehrungsvorgängen nachgewiesen.

Der oxydative Endabbau verläuft über cyanidempfindliche Fermente. Die Entkopplung der Phosphorylierungs-Vorgänge wird besonders bei proliferierenden Bakterien deutlich.

Malonsäure zeigt ein nicht zu erwartendes Verhalten. Es wird - besonders sichtbar bei endogener Atmung - von den Bakterien als Nährstoff aufgenommen.

Pyridin-3-sulfonat zeigt bei INH-sensiblen und -resistenten Keimen dem INH analoge Hemm effekte, so daß ein gemeinsamer Wirkungsmechanismus angenommen werden kann.

Die Fermente mit Thiolgruppen werden bei der sensiblen Variante stärker inhibiert als die del resistenten Bakterien. Weitere charakteristische Unterschiede zeigen sich in den Phosphorylierungsvorgängen und in der Hemmbarkeit des Citronensäurecyclus.
\end{abstract}

Ebenso wie auf anderen Gebieten der Medizin tritt auch bei der Chemotherapie der Tuberkulose das Resistenzproblem immer mehr in den Vordergrund. Besonders trifft dies für die Resistenz gegenüber Isonicotinsäurehydrazid (INH) zu. Die besonderen Eigenschaften INH-resistenter Mykobakterien, vor allem ihre im Tierversuch gegenüber Meerschweinchen verminderte Virulenz, waren Anlaß zu großen Hoffnungen in therapeutischer und epidemiologischer Hinsicht. Von experimenteller Seite wurde diesem Problem ebenfalls größte Aufmerksamkeit geschenkt. Mannigfache Unterschiede zwischen INH-sensiblen und -resistenten Keimen konnten nachgewiesen werden, so z. B. im Sauerstoffverbrauch ${ }^{1}$, in der Substratverwertung bei ruhenden und sich vermehrenden Zellen ${ }^{2,3}$, in der Aktivität verschiedener Fermente ${ }^{4-9}$, im Salicylateffekt und anderen ${ }^{10,11}$.

Angesichts der außerordentlichen Bedeutung dieses Problems für die praktische Tuberkulosebekämpfung und für die Grundlagenforschung erscheinen weitere

1 N. Nasta, Bull. Union int. Tuberculose XXVI, 306 [1956].

2 W. Käppler u. H. Iwainsky, Zbl. Bakteriol. Parasitenkunde, Infektionskrankh. Hyg. Abt. I, Orig. 174, 506 [1959].

3 H. Imainsky u. W. Käppler, Zbl. Bakteriol., Parasitenkunde, Infektionskrankh. Hyg. 176, 521 [1959].

4 G. Middlebrook, Amer. Rev. Tubercul. 69, 471 [1954].

5 A. Krebs, Beitr. Klin. Tubercul. 115, 460 [1956].

6 G. Meissner u. R. Bönicke, Beitr. Klin. Tubercul. 116, 501 [1957]. systematische Untersuchungen über den Aufbau und Stoffwechsel arzneimittelresistenter Mykobakterien unbedingt erforderlich. Es wird dabei nicht zu umgehen sein, die sensible Variante mit in die Versuche einzubeziehen.

In zahlreichen Arbeiten wurde z. B. über Fermente bzw. über sich aus ihrer Anwesenheit ableitende Stoffwechselreaktionen berichtet. Trotz der oft mühevollen und nicht ungefährlichen Untersuchungen läßt sich jedoch ein Uberblick über die wichtigsten Fermentsysteme noch nicht geben. Je nach der angewandten Bakterienart und dem benutzten Nährsubstrat differieren die Ergebnisse.

So konnte für einige Arten der Abbau der Kohlenhydrate nach EMBDEN-MEYeRHOF sichergestellt werden ${ }^{12}$, bei anderen Arten ist der "Glucose-Phosphat-Shunt" sicher mitbeteiligt ${ }^{13}$. Der Brenztraubensäure ist wegen der Verkettung des Kohlenhydrat-Abbaues mit dem des Glycerins und der Milchsäure eine zentrale Stellung

7 M. O. Tirunarayanan u. W. A. Vischer, Naturwissenschaften 43, 476 [1956].

8 A. Andrejew, A. Tacquet u. Ch. Gernez-Rieux, Ann. Inst. Pasteur 91, 586 [1956].

- R. BöNICKE, Naturwissenschaften 44, 619 [1957].

10 H. Pope, Amer. Rev. Tubercul. 69, 705 [1954].

11 R. BöNicke, Jber. Borstel 4, 127 [1956/57].

12 S. Faine, M. Whiteside u. N. L. Edson, Proc. Univ. Otago Med. School 29, 18 [1951].

13 I. C. Gunsalus, B. L. Horecker u. W. A. Wood, Bacteriol. Rev. 19, 79 [1955]. 
einzuräumen 14-17. Während eine Reihe von Lipasen und Esterasen beschrieben worden ist ${ }^{\mathbf{1 8 - 2 0}}$, konnten Fermente des oxydativen Abbaues von Fettsäuren bisher nicht isoliert werden, obwohl solche Fermentsysteme vorhanden sein müssen, wie die Verwendung von Fettsäuren als Kohlenstoffquelle ${ }^{21-23}$ beweist. Über den Umsatz stickstoffhaltiger Verbindungen ist wenig bekannt. Proteine werden kaum angegriffen ${ }^{24}$, dagegen läßt sich eine relativ starke Peptidasen-Aktivität nachweisen 25-27. Eine durch Streptomycin und INH hemmbare Diaminoxydase ist für die Bakteriengattung lebensnotwendig ${ }^{28-30}$. Weiter ist die Urease nachgewiesen worden ${ }^{31-33}$.

Die Zellmembran ist für Di- und Tricarbonsäuren impermeabel, verschiedentlich ist daraus ein Fehlen des Citronensäurecyclus abgeleitet worden ${ }^{34}$. Der oxydative Endabbau verläuft jedoch sehr wahrscheinlich über diesen Cyclus. Von den notwendigen Fermenten sind bereits eine Reihe beschrieben worden, so z. B. „Condensing“-Enzym, Aconitase, Isocitronensäuredehydrase, Succinoxydase ${ }^{35-37}$. Weiter lassen sich bei Anwendung von ${ }^{14}$ C-Acetat als Kohlenstoffquelle alle Glieder des Tricarbonsäurecyclus nachweisen ${ }^{38}$. Von Oxydo-Reduktasen sind u. a. Cytochrome ${ }^{39-43}$, Katalase ${ }^{44-48}$, Peroxydase ${ }^{49,50}$ sowie Milchsäuredehydrogenasen nachgewiesen worden.

Unsere Kenntnisse über die anabolischen Vorgänge sind sehr gering. Wenn auch anzunehmen ist, daß die meisten fermentativen Prozesse reversibel verlaufen können, so sind doch viele Schritte, insbesondere der Aufbau spezifischer Lipoidsubstanzen, wie Cordfaktor,

14 R. O. Loebel, E. Schorr u. H. B. Richardson, J. Bacteriol. 26, 139 [1933].

15 F. Bernheim, J. Bacteriol. 41, 387 [1941].

16 G. P. Youmans u. A. S. Youmans, J. Bacteriol. 65, 92, 100 [1953].

17 G. J. E. Hunter, Biochem. J. 55, 320 [1953].

18 K. Ogura, Sh. Imazu, M. Kato u. Y. Yamamura, Kekkaku 29, 128 [1954].

10 S. Cohen, J. B. Kushnik u. C. V. Purdy, J. Bacteriol. 66, 266 [1953].

20 C. Prina, Arch. Sci. med. [Paris] 96, 284 [1953].

21 W. Franke u. A. Schillinger, Biochem. Z. 316, 313 [1944].

22 W. Franke, Li-Tsoung u. D. Kibat, Biochem. Z. 319, 263 [1949].

23 M. Lindsay, T. V. O’Donnel u. N. L. Edson, Biochem. J. 46, 248 [1950].

24 K. G. Dernby u. C. Naeslund, Biochem. Z. 132, 393 [1922].

25 H. SAito, J. Biochemistry [Tokyo] 34, 223 [1941].

26 F. Roulet u. E. A. Zeller, Experientia [Basel] 1, 122 [1945].

27 M. N. Mickelson u. F. E. Shideman, J. Bacteriol. 51, 574 [1946].

28 F. Roulet u. E. A. Z Eller, Helv. chim. Acta 28, 1326 [1945].

29 C. A. Owen, E. A. Zeller u. G. A. Karlson, J. Bacteriol. 62, 53 [1951].

30 G. N. Perschin u. W. W. Nesswadba, Biochemie (russ.) 22, 804 [1957].

31 H. J. Corper u. H. C. Sweany, J. Bacteriol. 3, 129 [1918].

32 J. Singer u. E. Crsner, Amer. Rev. Tubercul. 65, 779 [1952].
Phthiokol, Mycolsäuren u. a. und die daran beteiligten Fermente noch unbekannt.

Das Ziel der vorliegenden Arbeit war es, einerseits durch Anwendung verschiedener Fermentinhibitoren unter Beschränkung auf die am häufigsten verwendete Kohlenstoffquelle Glycerin einen orientierenden Einblick in den Fermentapparat der Mykobakterien zu erhalten, zum anderen sollte nach weiteren Unterschieden im Stoffwechsel INH-sensibler und -resistenter Bakterien gesucht werden.

Eine Auftrennung nach unterschiedlichen Stoffwechsellagen (Untersuchungen unter Bedingungen der endogenen Atmung - ohne Substrat - ; der Ruheatmung - Zusatz einer Kohlenstoffquelle und der Vermehrungsatmung - Zusatz von Kohlenund Stickstoffquellen -) erschien sehr vorteilhaft. Naturgemäß lassen sich solche umfangreichen Versuchsreihen wegen der damit verbundenen Gefahren und experimentellen Schwierigkeiten kaum mit pathogenen Keimen durchführen. Daher wurde für die vorliegenden Untersuchungen $M$. phlei, ein säurefester Saprophyt, verwendet. $\mathrm{Zu}$ einer Übertragung der Versuchsergebnisse auf pathogene Vertreter dieser Bakteriengattung bedarf es aus bereits geschilderten Gründen gezielter Untersuchungen mit dem jeweiligen Stamm.

33 E. A. Zeller, L. van Orden u. W. Vögtli, J. biol. Chemistry 209, 429 [1953].

34 C. L. McLaren u. G. J. JanN, Amer. Rev. Tubercul. 71, 260 [1955].

35 I. Millman u. G. P. Youmans, J. Bacteriol. 69, 320 [1955].

36 M. Kusunose u. E. Kusunose, J. Biochemistry [Tokyo] 39, 34. [1952].

37 D. S. Goldman, J. Bacteriol. 73, 602 [1957]

38 A. S. Youmans, I. Millman u. G. P. Youmans, J. Bacteriol. 71, 565 [1956].

39 H. Yaor u. H. Tamija, J. Lab. clin. Med. 12, 795 [1927].

40 A. Fuijita u. T. Kodamama, Biochem. Z. 273, 186 [1934].

41 W. Frei, L. Riedmüller u. F. Almasy, Biochem. Z. 274, 253 [1934].

42 A. Andrejew u. H. I. Rosenberg, Bull. Soc. Chim. Biol. 34, 279 [1953].

43 A. Andrejew, C. R. Séances Soc. Biol. Filiales 148, 995 [1954].

44 M. HaHN, Münchener med. Wschr. 44, 1344 [1897].

45 E. R. Long, Amer. Rev. Tubercul. 3, 86 [1913].

46 M. K. Finlayson u. N. L. Edson, Proc. Roy. Soc. [New Zealand] 77, 284 [1949].

47 A. Andrejew, C. R. Séances Soc. Biol. Filiales 146, 650 [1952].

48 W. Uecker, Zbl. Bakteriol., Parasitenkunde, Infektionskrankh. Hyg., Abt. I, Orig. 164, 424 [1955].

$49 \mathrm{~W} . \mathrm{F}_{\mathrm{REI}}$, Zbl. Bakteriol., Parasitenkunde, Infektionskrankh., Abt. I, Orig. 134, 26 [1935].

50 G. Giolotri, Soc. Ital. Sci. Vet. 3, 547 [1949]. 


\section{Eingesetzte Inhibitoren}

Als Hemmstoffe wurden eingesetzt: Fluorid, Jodacetat, Arsenit, Fluoracetat, Malonat, Malonsäurediäthylester, Cyanid, Pyridin-3-sulfonat, 2.4-Dinitrophenol, Azid, Arsenat, Ninhydrin und Alloxan.

Hemmstoffe des Kohlenhydratabbaues: Fluorid hemmt in Form eines Magnesiumfluorphosphates die Enolase ${ }^{51,52}$. Weiter werden Bernsteinsäuredehydrogenase ${ }^{53}$, Carboxylase, Urease, verschiedene Phosphatasen ${ }^{54}$ und die Lipase von $M . t u b .{ }^{55}$ beeinflußt. Die Wirkung ist stark vom $p_{\mathrm{H}^{-}}$Wert abhängig. Die optimale Hemmung liegt etwa bei einem $p_{\mathrm{H}^{-}}$ Wert von 4,8 . Thiolenzyme werden im allgemeinen durch Jodacetat gehemmt. Im Fall des Kohlenhydrat-Abbaues ist speziell die Triosephosphatdehydrogenase ${ }^{56,57}$ betroffen, weiter werden beeinflußt Citronensäureoxydase, Äpfelsäuredehydrase und Milchsäureoxydase. Die Wirkung beider Inhibitoren auf die endogene und die Ruheatmung von Mykobakterien ist schon wiederholt beschrieben worden ${ }^{17,}$ 58-60.

Hemmstoffe des Citronensäurecyclus: Der wichtigste Angriffspunkt des Arsenits ist die Hemmung der oxydativen Pyruvatdecarboxylierung ${ }^{61}$. Ebenso wie im Falle des Jodacetats werden weitere Thiolenzyme inhibiert ${ }^{62}$, wie $z$. B. die Succinoxydase bei M. phlei. Die Wirkung der aus Fluoressigsäure gebildeten Fluorcitronensäure auf die Aconitase ${ }^{63}$ ist im Gegensatz zu den bisher aufgeführten Hemmstoffen sehr spezifisch. Außer der bekannten kompetitiven Hemmung der Bernsteinsäuredehydrogenase ${ }^{64}$ beeinflußt Malonsäure bzw. Malonsäurediäthylester

51 O. Warburg u. W. Christian, Naturwissenschaften 29, 590 [1941].

52 O. Warburg u. W. Christian, Biochem. Z. 311, 385 [1942].

53 E. C. Slater, Biochem. J. 58, i [1954].

54 L. Massart, Inhibitors of Enzyme, in: J. B. Sumner, K. Myrвӓск, „The Enzymes“, Volume I, Part I, S. 307 (1950) N. Y.

55 B. D. Davis u. R. J. Dubos, J. Bacteriol. 55, 11 [1948].

5o J. H. Quastel, Biochem. J. 27, 1116 [1933].

57 F. Dickens, Biochem. J. 27, 1141 [1933].

58 R. J. Fitzgerald u. F. Bernheim, J. Bacteriol. 55, 677 [1948].

59 A. Andrejew, C. R. Séances Soc. Biol. Filiales 145, 1630 [1951].

60 F. Bernheim, W. De Turk u. H. Pope, J. Bacteriol. 65, 544 [1953].

61 K. Rachamandran u. T. K. Walker, Biochem. J. 65, 20 [1957].

62 J. J. Gordon u. J. H. Quastel, Biochem. J. 42, 337 [1948].

63 R. A. Peters u. T. H. Wilson, Biochim. biophysica Acta [Amsterdam] 9, 310 [1952].

64 H. A. Krebs u. L. V. EgGleston, Biochem. J. 34, 1386 [1940]. auch die Fumarase ${ }^{65}$ und die Pyruvatdehydrogenase ${ }^{66}$.

Hemmstoffe der Atmungsfermente: Die Hemmung der oxydierten Form der Cytochromoxydase durch Cyanid ist bei Mykobakterien bereits untersucht worden ${ }^{67-69}$. Die in quantitativer Hinsicht abweichenden Ergebnisse sind wahrscheinlich durch Unterschiede im Kulturzustand und in der Anzüchtung zu erklären. Pyridin-3-sulfonat inhibiert die Milchsäuredehydrogenase ${ }^{70}$, die strukturelle Ähnlichkeit mit Nicotinsäureamid und Isonicotinsäurehydrazid sind im Zusammenhang mit den vorliegenden Problemen besonders interessant.

Entkoppler der Oxydations- und Phosphorylierungsvorgänge: Dinitrophenol entkoppelt die Atmungsketten-Phosphorylierung ${ }^{71}$, außerdem werden Beeinflussungen der Pyruvatdehydrogenase, der Diaminoxydase und der Carboxylase festgestellt ${ }^{54}$. Azid soll die Bildung von Diphosphoglycerinsäure aus 3-Phosphorglycerinaldehyd verhindern ${ }^{72}$. Auch Arsenat inhibiert die Bildung der 1.3-Diphosphoglycerinsäure $^{73}$. Nach KANDLER, Zehender und MüLler ${ }^{74}$ ist es ein universelles Phosphorylierungsgift, das Substrat- und Atmungsketten-Phosphorylierung hemmt.

Alloxan und Ninhydrin inhibieren Fermente mit Sulfhydrylgruppen, so z. B. Bernsteinsäuredehydrogenase $^{75}$, Diaminoxydase ${ }^{76}$ und andere mehr.

\section{Experimenteller Teil}

Arbeitsweise: Als Maß für die Stoffwechselleistungen wurde die Sauerstoffaufnahme mit Hilfe der $W_{\text {AR- }}$ BURG-Apparatur bestimmt ${ }^{77}$. Dazu wurden die Gefäße

${ }^{65}$ V. MASSEY, Biochem. J. 55, 320 [1953].

${ }^{66}$ E. Aubel u. J. Szulmajster, Biochim. biophysica. Acta [Amsterdam] 5, 255 [1950].

67 N. L. Edson u. G. J. E. Hunter, Biochem. J. 41, 139 [1947].

${ }^{68}$ A. Andrejew, C. R. Séances Soc. Biol. Filiales 143, 1443 [1949].

${ }^{69}$ Y. Yamamura u. Mitarb. J. Biochemistry [Tokyo] 41, 513 [1954].

70 H. v. Euler, Ber. dtsch. chem. Ges. 75, 1876 [1942].

71 W. F. Loomis u. F. Lipman, J. biol. Chemistry 173, 807 [1948].

72 S. Spiegelman, M. D. Kamen u. M. Sussman, Arch. Biochemistry 18, 409 [1948]

73 O. Warburg u. W. Christian, Biochem. Z. 303, 40 [1939].

74 O. Kandler, Cl. Zehender u. J. Müller, Arch. Mikrobiol. 24, 209 [1956].

75 F. G. Hopkins, E. J. Morgan u. C. Lutwak-Mann, Biochem. J. 32, 1829 [1938].

76 N. L. Edson, Bacteriol. Rev. 15, 147 [1951].

77 H. IWAinsky u. W. KäPpleR, Naturwissenschaften 43, 503 [1956]. 
mit je $2 \mathrm{ml}$ Bakteriensuspension beschickt. Der Einsatz enthielt 0,1 ml 20-proz. Kalilauge. Die Badtemperatur lag bei $37,5^{\circ} \mathrm{C}$, die Schüttelgeschwindigkeit betrug 180 Schwingungen/Min. bei einer Amplitude von 1 Zentimeter. Die Versuchsdauer war bei ruhenden Keimen (endogene und Ruheatmung) $6 \mathrm{Stdn}$.; bei sich vermehrenden Bakterien 12 Stunden. Die statistische Sicherung der Versuchsergebnisse erfolgte nach dem t-Verfahren ${ }^{78}$.

Verwendeter Bakterienstamm: Die Versuche wurden mit dem Stamm Mycobacterium phlei 169 „Berlin“ durchgeführt. Die Gründe für seine Auswahl, die Anzüchtung und die Vorbereitung der Versuchsansätze, sind bereits früher beschrieben worden ${ }^{79}$. Die Hemmdosis des INH liegt für die sensible Form bei 3 bis $4 \mu \mathrm{g} / \mathrm{ml}$ (fester Nährboden Hohn IV nach Got ts a cker). Die resistente Variante wurde in Gegenwart von $3000 \mu \mathrm{g}$ INH/ml subkultiviert. Um diese Resistenzhöhe zu erreichen, waren 30 Passagen notwendig. Die Katalase-Aktivität war bei sensiblen und resistenten Keimen gleich stark.

Verwendete Puffer bzw. Nährmedien: Als Suspensionsflüssigkeit diente für die endogene und die Ruheatmung 0,033-m. Phosphatpuffer nach $S$ ö re n s e n mit einem $p_{H}-$ Wert von 6,8. Für die Ansätze mit Fluorid wurde der $p_{\mathrm{H}^{-}}$Wert auf 4,8 eingestellt. Das Feuchtgewicht der Bakterien betrug jeweils $8-20 \mathrm{mg} / \mathrm{ml}$ (Trübungsmessung im $\mathrm{Pulf}$ rich-Photometer mit Filter L 53). Für die Messung der Ruheatmung wurde $30 \mathrm{Min}$. vor Versuchsbeginn $1 \mathrm{ml}$ des stickstofffreien Nährsubstrates (Glycerin 2,2-m.) und die auf einen $p_{\mathrm{H}^{-}}$

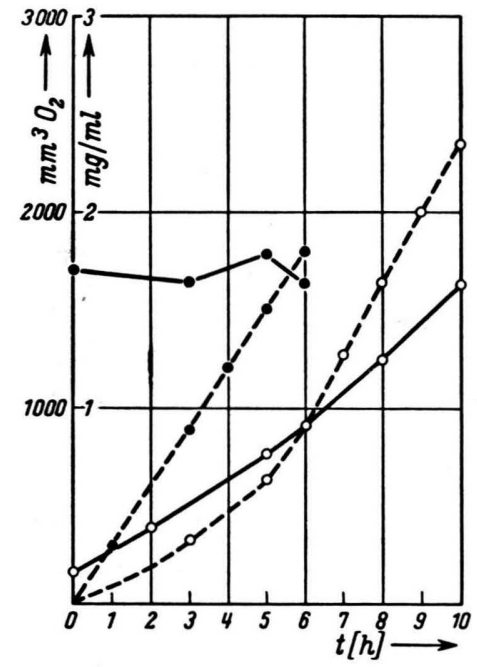

Abb. 1. Sauerstoffaufnahme und Trockengewicht (mg/ml) bei ruhenden und proliferierenden Zellen von $M$. phlei.

78 A. Linder, Statistische Methoden für Naturwissenschafter, Mediziner und Ingenieure, Birkhäuser Verlag, 2. Aufl., Basel 1951.

79 H. Iwainsky u. W. KäPpler, Zbl. Bakteriol., Parasitenkunde, Infektionskrankh., Hyg. Abt. I, Orig. 171, 178 [1958].

60 W. Herrmann, Z. Hyg. Infektionskrankh. 129, 146 [1946].

81 H. Iwainsky u. G. Müller, J. Hyg. Epid. Microbiol. Immunol., im Druck.

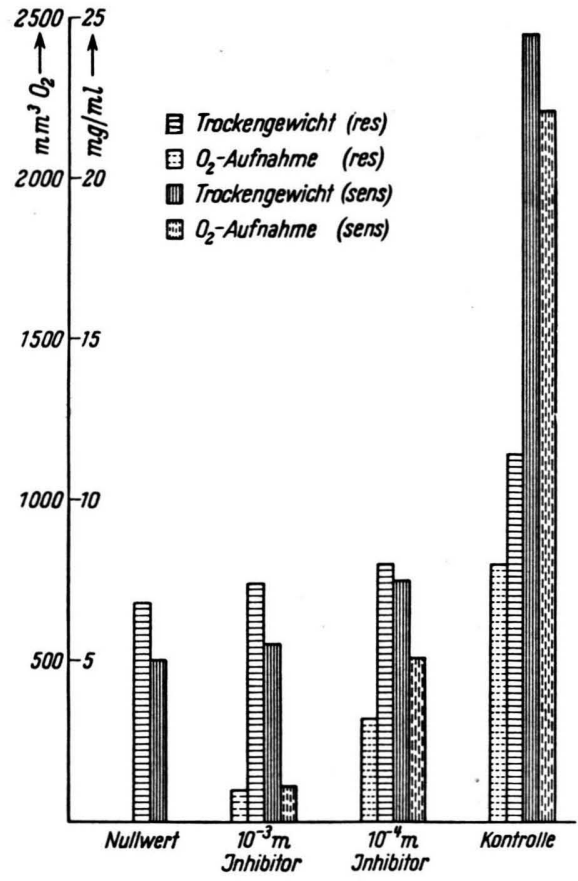

Abb. 2. Der Einfluß von Natriumarsenit auf Sauerstoffaufnahme und Trockengewicht bei proliferierenden INH-sens. und res. Zellen von $M$. phlei.

Wert von 6,8 eingestellte Lösung des Inhibitors zu $10 \mathrm{ml}$ der Bakteriensuspension zugesetzt. Der Ansatz enthielt außerdem $0,1 \%$ Tween 80 als Netzmittel.

Für die Vermehrungsatmung wurden $10 \mathrm{ml}$ einer sterilen, dem Ansatz der Ruheatmung analogen Suspension zu $100 \mathrm{ml}$ eines modifizierten $\mathrm{K}$ i r c h n e r - Substrates nach Herrmanv ${ }^{80}$ (ohne Malachitgrün, mit $0,05 \%$ Tween 80 ) zugegeben und 21 Stdn. im Brutschrank bei $37,5^{\circ} \mathrm{C}$ bebrütet. $30 \mathrm{Min}$. vor Versuchsbeginn wurden Glycerin (Endkonzentration 0,2-m.) und die jeweilige auf einen $p_{\mathrm{H}}$-Wert von 6,8 gebrachte sterile Hemmstofflösung zugesetzt. Das Feuchtgewicht lag bei diesen Ansätzen zwischen 0,5 bis $1,0 \mathrm{mg} / \mathrm{ml}$.

Sowohl bei den Versuchen mit ruhenden als auch mit proliferierenden Bakterien ließ sich die Proportionalität zwischen Substanzvermehrung und Sauerstoffaufnahme außer durch makroskopischen Vergleich durch Trockengewichts-Bestimmungen sichern (vgl. Abb. 1 und 2). Bei Vermehrungsvorgängen führt der Einbau von ${ }^{32} \mathrm{P}$ zu gleichen Ergebnissen ${ }^{81}$ (s. Abb. 3). Neben anderen Faktoren ändert sich die Wirkung verschiedener Inhibitoren in Abhängigkeit vom Bakterienalter. Um zu vergleichbaren Resultaten zu gelangen, wurden für alle Untersuchungen $3-5$ Tage alte Bakterienkulturen verwendet.

\section{Ergebnisse}

Endogene Atmung: Der Einfluß von Inhibitoren und ihrer Konzentration auf den Abbau zelleigener 
Substanzen - gemessen an der Sauerstoffaufnahme der Bakterien - ist in Tab. 1 wiedergegeben.

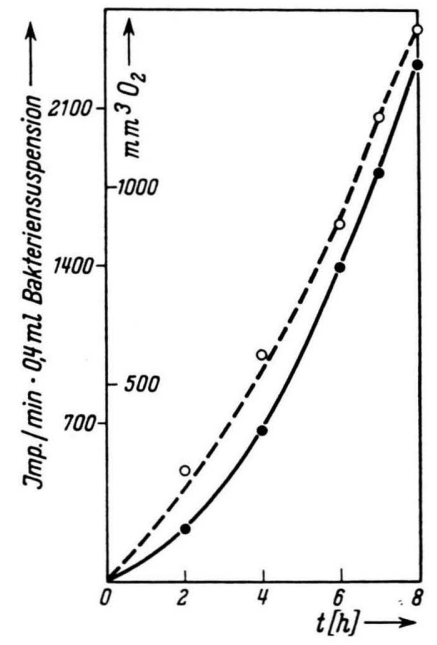

Abb. 3. Der Einbau von ${ }^{32} \mathrm{P}$ bei proliferierenden Zellen von M. phlei. (๑) : Sauerstoffaufnahme. $(0--0)$ : Aufnahme von ${ }^{32} \mathrm{P}$.
Danach wird die endogene Atmung durch Fluoracetat, 2.4-Dinitrophenol, Azid und Alloxan stimuliert, durch Fluorid bei einem $p_{\mathrm{H}}$-Wert von 4,8, Jodacetat, Cyanid, Arsenit, Malonsäurediäthylester und Ninhydrin zumindest in $10^{-2}-m$. Konzentration gehemmt, während im neutralen Bereich Fluorid ohne Wirkung ist. Malonsäure kann von den Bakterien als Nährsubstrat verwendet werden und führt daher naturgemäß zu einer Steigerung der Sauerstoffaufnahme. Ebenso wie INH ${ }^{* *}$ ist auch Pyridin-3-sulfonat in der angewendeten Konzentration ohne Einfluß auf die endogene Atmung, das gleiche gilt für Arsenat. Beim Zusatz von Jodacetat, Malonsäurediäthylester, Cyanid und Ninhydrin knickt die Sauerstoffaufnahme zumindest bei der höchsten geprüften Konzentration stark ab, während Arsenit eine etwa proportional gleichbleibende Beeinträchtigung zeigt. Eine unterschiedliche Wirkung auf INH-sensible und -resistente Bakterien zeigen Jodacetat und Azid.

Ruheatmung: Auf die Ruheatmung sind, wie aus Tab. 2 hervorgeht, Fluoracetat, Malonat, Dinitro-

\begin{tabular}{|c|c|c|c|c|c|c|c|}
\hline \multirow{2}{*}{ Hemmstoff } & \multirow{2}{*}{$\begin{array}{c}\text { Konzen- } \\
\text { tration } \\
{[-m .]}\end{array}$} & \multicolumn{2}{|c|}{ endogene Atmung } & \multirow{2}{*}{ Hemmstoff } & \multirow{2}{*}{$\begin{array}{c}\text { Konzen- } \\
\text { tration } \\
{[-m .]}\end{array}$} & \multicolumn{2}{|c|}{ endogene Atmung } \\
\hline & & sens. & res. & & & sens. & res. \\
\hline Fluorid & $\begin{array}{l}10^{-4} \\
10^{-3} \\
10^{-2}\end{array}$ & $\begin{array}{r}102 \\
98 \\
12\end{array}$ & & Jodacetat & $\begin{array}{l}10^{-4} \\
10^{-3} \\
10^{-2}\end{array}$ & $\begin{array}{c}109 \\
106 \\
59 *\end{array}$ & $\begin{array}{c}103 \\
91 \\
81\end{array}$ * \\
\hline Arsenit & $\begin{array}{l}10^{-4} \\
10^{-3} \\
10^{-2}\end{array}$ & $\begin{array}{r}103 \\
81 \\
76\end{array}$ & $\begin{array}{l}99 \\
74 \\
72\end{array}$ & Fluoracetat & $\begin{array}{l}10^{-4} \\
10^{-3} \\
10^{-2}\end{array}$ & $\begin{array}{l}109 \\
120 \\
119\end{array}$ & $\begin{array}{l}103 \\
108 \\
107\end{array}$ \\
\hline Malonat & $\begin{array}{l}10^{-3} \\
10^{-2} \\
10^{-1}\end{array}$ & $\begin{array}{l}143 \\
171 \\
176\end{array}$ & $\begin{array}{l}127 \\
183 \\
210\end{array}$ & Malonsäurediäthylester & $\begin{array}{l}10^{-3} \\
10^{-2} \\
10^{-1}\end{array}$ & $\begin{array}{r}157 \\
220 \\
62\end{array}$ & $\begin{array}{r}148 \\
210 \\
58\end{array}$ \\
\hline Cyanid & $\begin{array}{l}10^{-4} \\
10^{-3} \\
10^{-2}\end{array}$ & $\begin{array}{r}114 \\
69 \\
51\end{array}$ & $\begin{array}{r}115 \\
67 \\
58\end{array}$ & Pyridin-3-sulfonat & $\begin{array}{l}10^{-4} \\
10^{-3} \\
10^{-2}\end{array}$ & $\begin{array}{l}106 \\
108 \\
107\end{array}$ & $\begin{array}{l}102 \\
103 \\
107\end{array}$ \\
\hline 2.4-Dinitrophenol & $\begin{array}{l}10^{-5} \\
10^{-4} \\
10^{-3}\end{array}$ & $\begin{array}{l}101 \\
100 \\
128\end{array}$ & $\begin{array}{l}104 \\
109 \\
134\end{array}$ & Azid & $\begin{array}{l}10^{-4} \\
10^{-3} \\
10^{-2}\end{array}$ & $\begin{array}{c}99 \\
107 \\
138 *\end{array}$ & $\begin{array}{l}102 \\
101 \\
116\end{array}$ * \\
\hline Arsenat & $\begin{array}{l}10^{-4} \\
10^{-3} \\
10^{-2}\end{array}$ & $\begin{array}{r}98 \\
101 \\
101\end{array}$ & $\begin{array}{r}102 \\
99 \\
102\end{array}$ & Alloxan & $\begin{array}{l}10^{-4} \\
10^{-3} \\
10^{-2}\end{array}$ & $\begin{array}{l}105 \\
119 \\
181\end{array}$ & $\begin{array}{l}100 \\
104 \\
173\end{array}$ \\
\hline Ninhydrin & $\begin{array}{l}10^{-4} \\
10^{-3} \\
10^{-2}\end{array}$ & $\begin{array}{r}109 \\
93 \\
15\end{array}$ & $\begin{array}{l}97 \\
93 \\
15\end{array}$ & & & & \\
\hline
\end{tabular}

Tab. 1. Veränderungen der endogenen Atmung von INH-sensiblen und -resistenten Mykobakterien unter dem Einfluß verschiedener Inhibitoren.

* Die Unterschiede zwischen den Werten für die res. und sens. Keime sind statistisch gesichert $(P<0,01)$. Die Zahlenwerte bedeuten Prozente der Sauerstoffaufnahme der Kontrollen.

** Versuchsergebnisse und methodische Einzelheiten siehe G. MüLlER, „Physiologisch-chemische Untersuchungen am INH-sensiblen und INH-resistenten Mycobacterium phlei
169.“ Inaugural-Dissertation, Math.-nat. Fakultät, Humboldt-Universität zu Berlin 1959. 


\begin{tabular}{|c|c|c|c|c|c|c|c|}
\hline \multirow{2}{*}{ Hemmstoff } & \multirow{2}{*}{$\begin{array}{c}\text { Konzen- } \\
\text { tration } \\
{[-m .]}\end{array}$} & \multicolumn{2}{|c|}{ Ruheatmung } & \multirow{2}{*}{ Hemmstoff } & \multirow{2}{*}{$\begin{array}{c}\text { Konzen- } \\
\text { tration } \\
{[-m .]}\end{array}$} & \multicolumn{2}{|c|}{ Ruheatmung } \\
\hline & & sens. & res. & & & sens. & res. \\
\hline Fluorid & $\begin{array}{l}10^{-4} \\
10^{-3} \\
10^{-2}\end{array}$ & $\begin{array}{l}93 \\
95 \\
95\end{array}$ & $\begin{array}{l}101 \\
100 \\
103\end{array}$ & Jodacetat & $\begin{array}{l}10^{-4} \\
10^{-3} \\
10^{-2}\end{array}$ & $\begin{array}{l}91 \\
42 \\
18 *\end{array}$ & $\begin{array}{l}93 \\
58 \\
31\end{array}$ \\
\hline Arsenit & $\begin{array}{l}10^{-4} \\
10^{-3} \\
10^{-2}\end{array}$ & $\begin{array}{l}81 \\
42 \\
31\end{array}$ & $\begin{array}{l}81 \\
56 \\
30\end{array}$ & Fluoracetat & $\begin{array}{l}10^{-4} \\
10^{-3} \\
10^{-2}\end{array}$ & $\begin{array}{r}104 \\
102 \\
96\end{array}$ & $\begin{array}{r}100 \\
100 \\
97\end{array}$ \\
\hline Malonat & $\begin{array}{l}10^{-3} \\
10^{-2} \\
10^{-1}\end{array}$ & $\begin{array}{l}112 \\
112 \\
102\end{array}$ & $\begin{array}{r}102 \\
99 \\
97\end{array}$ & Malonsäurediäthylester & $\begin{array}{l}10^{-3} \\
10^{-2} \\
10^{-1}\end{array}$ & $\begin{array}{r}114 \\
121 \\
39\end{array}$ & $\begin{array}{r}107 \\
113 \\
34\end{array}$ \\
\hline Cyanid & $\begin{array}{l}10^{-4} \\
10^{-3} \\
10^{-2}\end{array}$ & $\begin{array}{l}58 \\
13 \\
12\end{array}$ & $\begin{array}{r}66 \\
13 \\
9\end{array}$ & Pyridin-3-sulfonat & $\begin{array}{l}10^{-4} \\
10^{-3} \\
10^{-2}\end{array}$ & $\begin{array}{r}101 \\
97 \\
77\end{array}$ & $\begin{array}{l}98 \\
98 \\
78\end{array}$ \\
\hline 2.4-Dinitrophenol & $\begin{array}{l}10^{-5} \\
10^{-4} \\
10^{-3}\end{array}$ & $\begin{array}{l}100 \\
115 \\
106\end{array}$ & $\begin{array}{r}104 \\
109 \\
93\end{array}$ & Azid & $\begin{array}{l}10^{-4} \\
10^{-3} \\
10^{-2}\end{array}$ & $\begin{array}{c}97 \\
98 \\
103\end{array}$ * & $\begin{array}{c}100 \\
103 \\
71\end{array}$ * \\
\hline Arsenat & $\begin{array}{l}10^{-4} \\
10^{-3} \\
10^{-2}\end{array}$ & $\begin{array}{c}102 \\
84 * \\
81 *\end{array}$ & $\begin{array}{r}103 \\
102 * \\
99 *\end{array}$ & Alloxan & $\begin{array}{l}10^{-4} \\
10^{-3} \\
10^{-2}\end{array}$ & $\begin{array}{l}100 \\
102 \\
127\end{array}$ & $\begin{array}{l}102 \\
109 \\
135\end{array}$ \\
\hline Ninhydrin & $\begin{array}{l}10^{-4} \\
10^{-3} \\
10^{-2}\end{array}$ & $\begin{array}{r}99 \\
33 \\
6\end{array}$ & $\begin{array}{r}99 \\
36 \\
3\end{array}$ & & & & \\
\hline
\end{tabular}

Tab. 2. Hemmung der Ruheatmung von INH-sensiblen und -resistenten Mykobakterien unter dem Einfluß verschiedener Inhibitoren.

* Die Unterschiede zwischen den Werten für die res. und sens. Keime sind statistisch gesichert $(P<0,01)$. Die Zahlenwerte bedeuten Prozente der Sauerstoffaufnahme der Kontrollen.

phenol und Alloxan ohne Wirkung. Ein plötzliches Absinken der Sauerstoffaufnahme ist nach Zusatz von Cyanid festzustellen. Bei der Anwendung von Jodacetat, Arsenit, Pyridin-3-sulfonat, Arsenat (nur sensible Keime) und Ninhydrin ist eine der Arsenitwirkung bei der endogenen Atmung entsprechende Beeinflussung der Sauerstoffaufnahme nachzuweisen. Eine stärkere Beeinträchtigung der INH-sensiblen Keime zeigt sich bei den Versuchen mit Jodacetat, Arsenat und Ninhydrin; Azid ist ohne Einfluß auf die Sauerstoffaufnahme der sensiblen Keime, die der resistenten dagegen ist partiell inhibiert.

Vermehrungsatmung: Fluorid kann als Inhibitor für Vermehrungsvorgänge bei dem $p_{\mathrm{H}}$-Wert seines Wirkungsoptimums $\left(p_{\mathrm{H}}\right.$-Wert 7,8$)$ nicht eingesetzt werden. Bei diesem $p_{\mathrm{H}^{-}}$Wert wird die Vermehrung von Mykobakterien praktisch unterbunden ${ }^{2,82}$. Malonat und Alloxan sind ohne Wirkung auf die Vermehrungsatmung.

Die übrigen Inhibitoren lassen sich drei verschiedenen Gruppen zuordnen.

\footnotetext{
82 J. Meissner, Z. Hyg. 145, 42 [1958].
}

Bei der ersten Gruppe ist verzögerte Vermehrung nachzuweisen. Die Wirkungssteigerung bei Erhö-

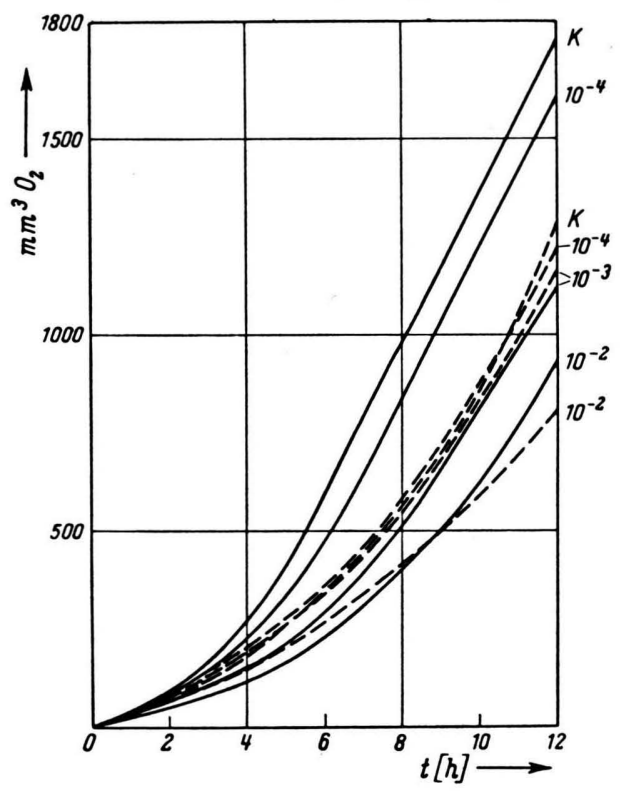

Abb. 4. Der Einfluß von Fluoracetat auf die Sauerstoffaufnahme proliferierender INH-sens. und -res. Mykobakterien. $(\longrightarrow)$ : sensible Keime. $(---)$ : resistente Keime. 


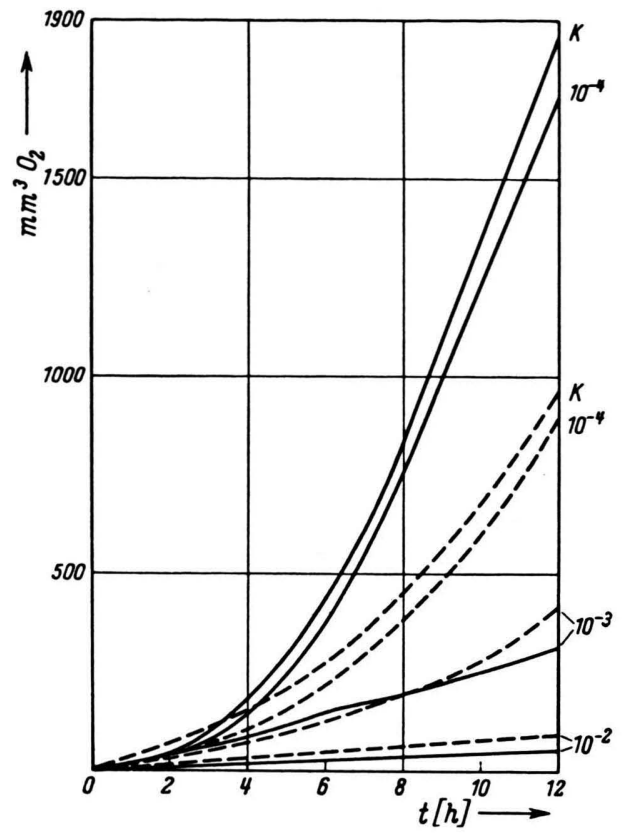

Abb.5. Der Einfluß von Jodacetat auf die Sauerstoffaufnahme proliferierender INH-sens. und -res. Mykobakterien. $(\stackrel{-}{-}$ : sensible Keime. $(---)$ : resistente Keime.

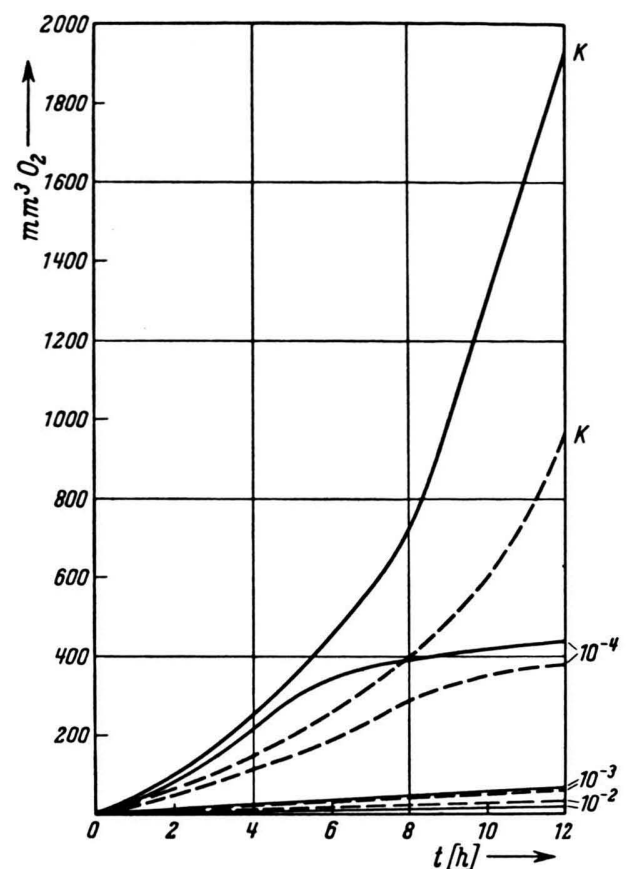

Abb. 6. Der Einfluß von Cyanid auf die Sauerstoffaufnahme proliferierender INH-sens. und -res. Mykobakterien.

$(\stackrel{-}{-}$ : sensible Keime. $(---)$ : resistente Keime.

\begin{tabular}{|c|c|c|c|c|c|c|c|}
\hline \multirow{2}{*}{ Hemmstoff } & \multirow{2}{*}{$\begin{array}{c}\text { Konzen- } \\
\text { tration } \\
{[-m .]}\end{array}$} & \multicolumn{2}{|c|}{$\begin{array}{l}\text { Vermehrungs- } \\
\text { atmung }\end{array}$} & \multirow[t]{2}{*}{ Hemmstoff } & \multirow{2}{*}{$\begin{array}{c}\text { Konzen- } \\
\text { tration } \\
{[-m .]}\end{array}$} & \multicolumn{2}{|c|}{$\begin{array}{l}\text { Vermehrungs- } \\
\text { atmung }\end{array}$} \\
\hline & & sens. & res. & & & sens. & res. \\
\hline Fluorid & $\begin{array}{l}10^{-4} \\
10^{-3} \\
10^{-2}\end{array}$ & $\begin{array}{l}98 \\
96 \\
93\end{array}$ & $\begin{array}{l}99 \\
92 \\
91\end{array}$ & Jodacetat & $\begin{array}{l}10^{-4} \\
10^{-3} \\
10^{-2}\end{array}$ & $\begin{array}{l}91 \\
16 * \\
5 *\end{array}$ & $\begin{array}{l}93 \\
40 * \\
13\end{array}$ \\
\hline Arsenit & $\begin{array}{l}10^{-5} \\
10^{-4} \\
10^{-3} \\
10^{-2}\end{array}$ & $\begin{array}{l}95 \\
44 \\
11 \\
2 *\end{array}$ & $\begin{array}{c}96 \\
39 \\
9 \\
6\end{array}$ & Fluoracetat & $\begin{array}{l}10^{-4} \\
10^{-3} \\
10^{-2}\end{array}$ & $\begin{array}{l}90 \\
65^{*} \\
53^{*}\end{array}$ & $\begin{array}{l}95 \\
88 * \\
61 \text { * }\end{array}$ \\
\hline Malonat & $\begin{array}{l}10^{-3} \\
10^{-2} \\
10^{-1}\end{array}$ & $\begin{array}{r}102 \\
97 \\
93\end{array}$ & $\begin{array}{r}103 \\
97 \\
86\end{array}$ & Malonsäurediäthylester & $\begin{array}{l}10^{-3} \\
10^{-2} \\
10^{-1}\end{array}$ & $\begin{array}{r}104 \\
107 \\
11\end{array}$ & $\begin{array}{r}115 \\
123 \\
13\end{array}$ \\
\hline Cyanid & $\begin{array}{l}10^{-4} \\
10^{-3} \\
10^{-2}\end{array}$ & $\begin{array}{c}22 \\
3 * \\
2 *\end{array}$ & $\begin{array}{r}24 \\
6 * \\
4^{*}\end{array}$ & Pyridin-3-sulfonat & $\begin{array}{l}10^{-4} \\
10^{-3} \\
10^{-2}\end{array}$ & $\begin{array}{r}100 \\
15 * \\
1 *\end{array}$ & $\begin{array}{l}99 \\
48^{*} \\
3^{*}\end{array}$ \\
\hline 2.4-Dinitrophenol & $\begin{array}{l}10^{-5} \\
10^{-4} \\
10^{-3}\end{array}$ & $\begin{array}{r}104 \\
103 \\
32\end{array}$ & $\begin{array}{r}102 \\
93 \\
38\end{array}$ & Azid & $\begin{array}{l}10^{-4} \\
10^{-3} \\
10^{-2}\end{array}$ & $\begin{array}{l}88 \\
68 * \\
24 *\end{array}$ & $\begin{array}{l}94 \\
47^{*} \\
17\end{array}$ \\
\hline Arsenat & $\begin{array}{l}10^{-4} \\
10^{-3} \\
10^{-2}\end{array}$ & $\begin{array}{l}113 \\
74 * \\
41 *\end{array}$ & $\begin{array}{r}103 \\
100 * \\
54 *\end{array}$ & Alloxan & $\begin{array}{l}10^{-4} \\
10^{-3} \\
10^{-2}\end{array}$ & $\begin{array}{l}101 \\
100 \\
105\end{array}$ & $\begin{array}{r}103 \\
100 \\
99\end{array}$ \\
\hline Ninhydrin & $\begin{array}{l}10^{-4} \\
10^{-3} \\
10^{-2}\end{array}$ & $\begin{array}{c}96 \\
45 \\
4\end{array}$ & $\begin{array}{c}98 \\
47 \\
9 *\end{array}$ & & & & \\
\hline
\end{tabular}

Tab. 3. Hemmung der Vermehrungsatmung von INH-sensiblen und -resistenten Mykobakterien unter dem Einfluß verschiedener Inhibitoren.

* Die Unterschiede zwischen den Werten für die res. und sens. Keime sind statistisch gesichert $(P<0,01)$. Die Zahlenwerte bedeuten Prozente der Sauerstoffaufnahme der Kontrollen. 
hung der Hemmstoffkonzentration ist relativ gering (vgl. Abb. 4).

Eine mit steigender Inhibitormenge rasch ansteigende Hemmung kennzeichnet die zweite Gruppe. Die bei niederen Konzentrationen verzögerte Vermehrung wird schließlich völlig unterbunden. Bei partieller Hemmung wird die dann meßbare Sauerstoffaufnahme praktisch nicht beeinflußt (s. Abb. 5). Die Wirkung dieser beiden Gruppen dürfte annähernd einer kompetitiven Hemmung entsprechen.

Die Sauerstoffaufnahme läuft in der dritten Gruppe bis zu einigen Stdn. mit den Kontrollen konform, um dann rasch abzusinken. Wie aus Abb. 6 hervorgeht, wird bei Inhibitoren dieser Gruppe auch die Sauerstoffaufnahme der nicht mehr vermehrungsfähigen Keime in Mitleidenschaft gezogen. Als Wirkungsmechanismus ist hier eine nicht kompetitive Hemmung anzunehmen. Dem erstgenannten Wirkungstyp sind Fluoracetat und Arsenat zuzuordnen. Die Wirkung von Jodacetat, Arsenit, Dinitrophenol, Azid und Ninhydrin entsprechen der in Abb. 5 dargestellten Wirkung der zweiten Gruppe. Cyanid und Pyridin-3-sulfonat zeigen eine der dritten Gruppen entsprechende Hemmung.

Bei Anwendung von Jodacetat, Fluoracetat, Pyridin-3-sulfonat, Arsenat und Azid ist eine unterschiedliche Beeinflussung zwischen INH-sensiblen und -resistenten Keimen nachweisbar. Die ersten vier Inhibitoren wirken auf die sensiblen Keime stärker als auf die resistenten, Azid zeigt dagegen eine umgekehrte Wirkung.

\section{Diskussion der Ergebnisse}

Endogene und Ruheatmung: Bei der Beurteilung von Ganzzellversuchen mit Mykobakterien stellt bekanntlich die Permeabilitäts-Schranke einen experimentell vorerst noch nicht auszuschaltenden Unsicherheitsfaktor dar. Trotzdem kann auf Grund der Wirkung von Fluorid, Jodacetat und Arsenit für den Abbau zelleigener Reservestoffe der gleiche Weg angenommen werden, wie er von Hunter ${ }^{17}$ für die Dissimilation des Glycerins über Glycerophosphat, Triosephosphat, Phosphoglycerinsäure, Phosphoenolbrenztraubensäure, Brenztraubensäure zu „aktivierter Essigsäure“ und Kohlendioxyd vorgeschla-

83 H. Bцoch, Schweiz. Z. Pathol. 7, 589 [1944].

84 F. N. Stokes u. J. R. Campbell, Arch. Biochemistry 30, 121 [1950]. gen wurde. Damit können die Ergebnisse von AndRejew ${ }^{59}$, Bernheim u. anderen ${ }^{60}$ sowie Bloch ${ }^{83}$, bestätigt werden, soweit die experimentellen Bedingungen vergleichbar sind. Besonders trifft dies für den Einfluß des $p_{\mathrm{H}^{-}}$-Wertes auf die Fluoridhemmung $\mathrm{zu}^{76,84}$. Die mit dem skizzierten Reaktionsablauf gekoppelten Phosphorylierungs-Vorgänge lassen sich durch Zusatz von Dinitrophenol und Azid nachweisen. Die eintretende Erhöhung des Sauerstoffverbrauchs entspricht den in der Literatur bekanntgegebenen Befunden. Die Bedeutung der bereits nachgewiesenen Cytochrome ${ }^{17,67-69}$ für den oxydativen Endabbau wird durch die starke, zum Zelltod führende Inhibition durch Cyanid gezeigt.

Die Rolle des Citronensäurecyclus als Zwischenglied ist nach den vorliegenden Untersuchungen noch unklar. Der Zusatz von Fluoracetat zeigt nicht den erwarteten Effekt. Malonat wird ebenso wie wahrscheinlich Alloxan als Substrat verwendet und scheidet daher als Inhibitor aus. Die starke aber wenig spezifische Hemmung durch Ninhydrin zeigt die Bedeutung von Thiolenzymen an.

Unter Bedingungen der Ruheatmung ergeben sich hinsichtlich des Abbauweges des Glycerins, der Rolle des Citronensäurecyclus und des oxydativen Endabbaues analoge Ergebnisse. Pyridin-3-sulfonat hemmt den oxydativen Abbau des Glycerins ähnlich wie INH, wobei ein Einfluß der Glycerinkonzentration deutlich festzustellen ist ${ }^{85}$.

Vermehrungsatmung: Bei proliferierenden Mykobakterien sind wahrscheinlich beim Aufbau der Zellsubstanzen eine Reihe von Fermenten beteiligt, die für die endogene und die Ruheatmung nicht beansprucht werden. Damit findet die sowohl an Zahl der Inhibitoren als auch hinsichtlich ihrer Konzentration festgestellte Wirkungssteigerung ihre Erklärung. Unterschiede zu den Versuchen bei endogener und Ruheatmung können sich anderseits durch die notwendige Verlängerung der Versuchsdauer bei der Keimvermehrung ergeben. Weiter ist zu berücksichtigen, daß das verwendete Nährsubstrat (Kirchner-Substrat 30) unter Umständen den Bakterien nach partieller oder totaler Ausschaltung des Glycerins ein Ausweichen auf andere Kohlenstoff- oder Stickstoffquellen ermöglicht.

85 W. Tysarowski, Bull. Acad. Polonaise Sci. Cl. II-Vol. II, 4,141 [1954].

86 H. L. Kornberg u. H. A. Krebs, Nature [London] 179, 988 [1957]. 
Aus den Versuchen ergibt sich, daß der Metabolismus des Glycerins bis zur oxydativen Decarboxylierung der Brenztraubensäure in der gleichen Weise abläuft wie bisher beschrieben. Im Gegensatz zur endogenen und der Ruheatmung ist jedoch, wie die Wirkung des Fluoracetats zeigt, die Beteiligung des Citronensäurecyclus für ein ungehindertes Wachstum notwendig. Seine Blockierung führt aber nicht zum Vermehrungsstop. Als weiteres mögliches Zwischenglied zwischen dem Embden-M e yer h of Schema und dem oxydativen Endabbau wäre der „Glyoxylsäurecyclus“ " 86 zu diskutieren, der, wie aus den Versuchen zu folgern ist, wahrscheinlich sogar den in quantitativer Hinsicht bedeutungsvolleren Abbauweg darstellt. In dieses Schema läßt sich aber das Verhalten der Malonsäure als Inhibitor nicht einordnen. Ihre nachgewiesene Verwendung als Nährstoff deutet die besonderen Stoffwechseleigenarten der Mykobakterien an. Bei weiteren Untersuchungen ist diesem Problem besondere Aufmerksamkeit zu widmen.

Der oxydative Endabbau verläuft, wie zu erwarten, ebenfalls wieder über die mit Cyanid hemmbaren Fermente. Das sehr ähnliche Wirkungsbild des Pyridin-3-sulfonats und des INH läßt auf die Blockierung eines gleichartigen für die Vermehrungsvorgänge wichtigen Fermentes oder Fermentsystems schließen. Eine Entkopplung der Phosphorylierung führt naturgemäß zu einer Inhibition der Vermehrungsvorgänge. In gleicher Weise wie Fluoressigsäure wirkt der Zusatz von Arsenat. Eine befriedigende Erklärung für den Angriffspunkt dieses Inhibitors steht aber im vorliegenden Falle noch aus.

Ein zusammenfassender Überblick über die mit Hilfe der eingesetzten Inhibitoren festgestellten fermentativen Prozesse bei $M$. phlei ergibt folgendes: Der Abbau zelleigener Reservestoffe verläuft ebenso wie der Metabolismus des Glycerins bei ruhenden und proliferierenden Bakterien zunächst nach dem E m b d e n - M e y e r h of - Schema. Der weitere Weg bis zum oxydativen Endabbau über die Cytochrome ist noch nicht geklärt. Es ist anzunehmen, daß er über den sog. "Glyoxylsäurecyclus“ verläuft. Eine Beteiligung des Citronensäurecyclus ist aber zumindest bei Vermehrungsvorgängen mit Sicherheit nachweisbar. Um weitere Einblicke zu erhalten, ist besonders die Rolle der Malonsäure eingehend zu untersuchen.
Vergleich des Fermenthaushaltes INH-sensibler und -resistenter

$$
\text { Zellen von } M \text {. phlei }
$$

Aus der etwa gleich großen Sauerstoffaufnahme bei INH-sensiblen und -resistenten Keimen bei der endogenen und der Ruheatmung bei der Verwendung von Glycerin als Kohlenstoffquelle wäre auf einen auch quantitativ gleichartigen Fermentapparat zu schließen. Ebenso wie beim Einsatz verschiedener Inhibitoren zeigen sich aber auch bei der Messung der Vermehrungsatmung recht charakteristische Unterschiede. Die pro Zeiteinheit aufgenommenen Sauerstoffmengen sind im Vergleich zu den sensiblen Keimen bei der resistenten Variante wesentlich vermindert. Der Kurvenverlauf entspricht einer Wachstumsverzögerung.

Eindrucksvolle Unterschiede im Fermentsystem beider Varianten lassen sich bei der Anwendung von Jodacetat, Arsenat und Azid feststellen. So wird z. B. die Sauerstoffaufnahme der sensiblen Zellen bei allen drei untersuchten Stoffwechsellagen durch den Zusatz von Jodacetat stärker inhibiert als die der resistenten. Demnach scheinen die resistenten Zellen die Möglichkeit zu besitzen, der Inhibierung der Thiolenzyme, speziell der Triosephosphatdehydrogenase, durch verstärkte Inanspruchnahme anderer Stoffwechselsysteme zu begegnen. Auch die auf sensible und resistente Keime unterschiedliche Wirkung von Ninhydrin kann im gleichen Sinn gedeutet werden.

Die sensiblen Keime zeigen weiter sowohl in der Ruhe- als auch in der Vermehrungsatmung nach $\mathrm{Zu}$ satz von Arsenat eine stärkere Beeinflussung als die resistente Variante. Bei der Verwendung von Azid als Inhibitor sind dagegen die größeren Hemmeffekte bei den resistenten Keimen nachzuweisen. Daraus ist zu folgern, daß mit der Resistenzentwicklung gegenüber INH eine Veränderung der Phosphorylierungs-Vorgänge gekoppelt ist. Die resistenten Keime zeigen darüberhinaus eine Beeinträchtigung des Citronensäurecyclus.

Pyridin-3-sulfonat wirkt auf INH-sensible und -resistente Keime in analoger Weise wie INH (vgl. Abb. 7 und 8). Damit sind möglicherweise Anhaltspunkte für die Wirkungsweise dieses Tuberkulostatikums gegeben. Auf Grund der engen strukturellen Beziehungen zu Nicotinsäureamid kann ein gegenseitiger Austausch in den Codehydrogenasen angenommen werden, wie er für INH bereits in 


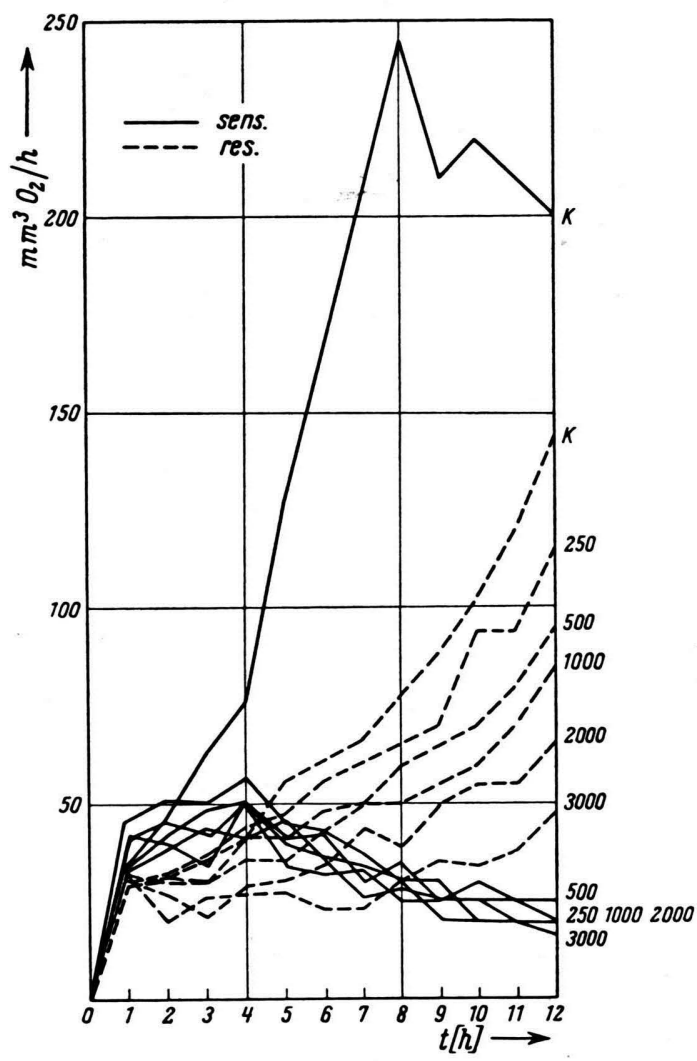

Abb. 7. Der Einfluß von INH auf die stündliche Sauerstoffaufnahme proliferierender Zellen von $M$. phlei. $(250,500 \ldots=250 \mu \mathrm{g}, 500 \mu \mathrm{g} \ldots$ Inhibitor $/ \mathrm{ml})$.

vitro aufgezeigt worden ist ${ }^{87,88}$. Die Hemmung der Vermehrungsvorgänge dürfte dann durch Fehlen der bei der Atmungsketten-Phosphorylierung gebildeten energiereichen Phosphatverbindungen bedingt sein. Eine Inhibierung der Milchsäuredehydrogenase

87 E. C. G. Hofmann u. S. Rapoport, Biochem. Z. 329, 437 [1958].

88 L. J. Zatman u. Mitarb., J. biol. Chemistry 209, 453, 467 [1954].

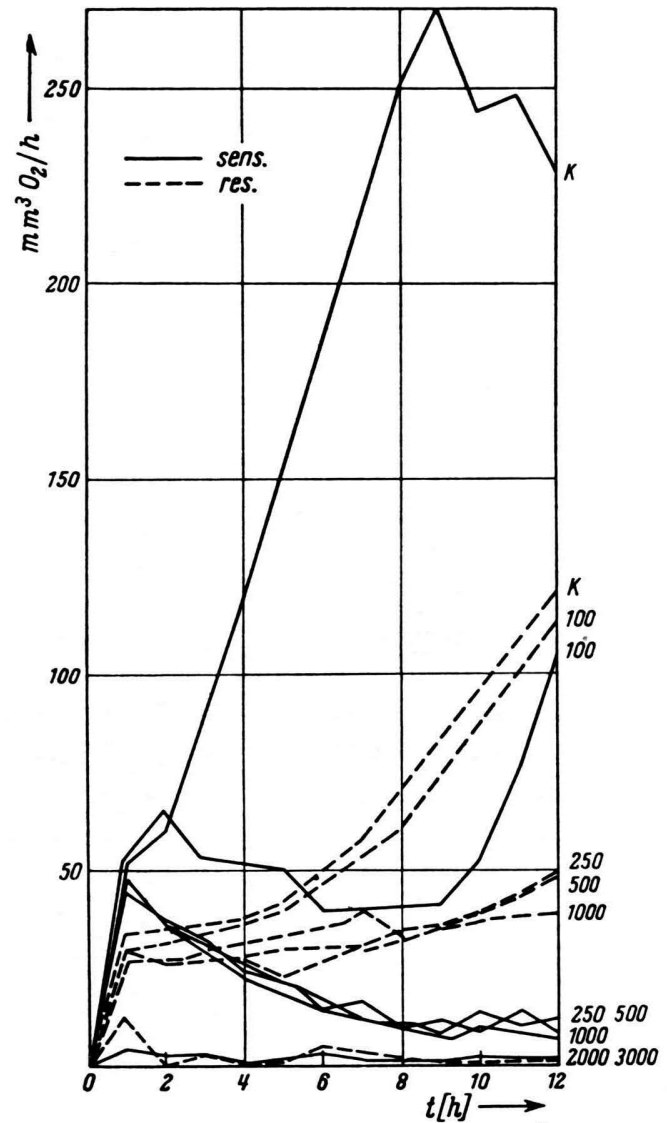

Abb. 8. Der Einfluß von Pyridin-3-sulfonat auf die stündliche Sauerstoffaufnahme proliferierender Zellen von $M$. phlei. $(250,500 \ldots=250 \mu \mathrm{g}, 500 \mu \mathrm{g} \ldots$. Inhibitor $/ \mathrm{ml})$.

durch Pyridin-3-sulfonat erscheint dagegen wegen der bei der endogenen und Ruheatmung ausbleibenden Wirkung wenig wahrscheinlich.

Herrn Dr. W. KäPPLER sind wir für die Überlassung der Bakterienstämme und für die Durchführung bakteriologischer Untersuchungen sehr zu Dank verpflichtet. Frau H. Kluge, Frau I. Stephan und Herre G. Hoinkis sei auch an dieser Stelle für ihre eifrige und gewissenhafte Mitarbeit herzlich gedankt. 